\title{
Negative prospective memory: Remembering not to perform an action
}

\author{
Jeffrey E. Pink • Chad S. Dodson
}

Published online: 7 November 2012

(C) Psychonomic Society, Inc. 2012

\begin{abstract}
Sometimes it is important to remember not to perform an action, such as remembering to stop taking seasonal allergy medicine when it is no longer needed. Mistakes in accomplishing this goal can involve prospective memory commission errors when individuals mistakenly perform a prospective response. In two experiments, we investigated the role of attentional resources in preventing prospective memory errors to cues that had been associated with a habitual prospective response. In Phase 1 of our experiments, participants performed a prospective memory task during which they either routinely responded to prospective memory cues or responded to these cues one time only. On a subsequent prospective memory task, the participants who had routinely responded to prospective memory cues were vulnerable to commission errors when their attentional resources were taxed. By contrast, dividing attention did not increase commission errors to cues that had not been routinely performed on an earlier task. These data indicate that attentional resources are needed to withhold making habitual prospective memory responses, and they suggest that inhibitory failures are a cause of these prospective memory errors. Finally, we suggest a broader definition of prospective memory that includes remembering to withhold performing actions.
\end{abstract}

Keywords Prospective memory $\cdot$ Memory and attention . False memory

Prospective memory refers to memory for intended actions that need to be completed in the future, such as remembering to tell a colleague an important piece of information

J. E. Pink · C. S. Dodson $(\bowtie)$

Department of Psychology, University of Virginia,

Charlottesville, VA 22904-4400, USA

e-mail: cdodson@virginia.edu
(Einstein \& McDaniel, 1990). We are investigating a form of prospective memory that we refer to as negative prospective memory, which we define as remembering not to perform an action when encountering a particular cue. For example, often one must remember not to do certain actions, such as remembering not to tell a colleague about a secret (e.g., a surprise party or hiring decision) or remembering not to take seasonal allergy medication when the allergy season is over. In many situations, one must withhold performing an action when encountering a relevant cue. However, we suspect that individuals occasionally have difficulty withholding prospective responses, particularly when the prospective responses have been performed repeatedly.

Why should it be difficult to inhibit performing certain actions, particularly intended actions that have already been completed (i.e., they are finished) and are no longer required? In fact, some evidence has suggested that inhibitory failures should play no role in the occurrence of prospective memory errors in response to cues that are associated with completed intentions (e.g., Marsh, Hicks, \& Bink, 1998; Marsh, Hicks, \& Bryan, 1999; Scullin, Einstein, \& McDaniel, 2009). Consider, for example, the "intention interference effect" - the finding that individuals are slower to make lexical-decision (i.e., word/nonword) judgments when they encounter prospective cues that are associated with an intended response, relative to words with no associated intention (e.g., Cohen, Dixon, \& Lindsay, 2005; Einstein et al., 2005; Marsh, Hicks, \& Watson, 2002). Scullin et al. (2009) showed that the intention interference effect disappears when prospective cues are associated with finished intentions, indicating that finished intentions are deactivated and no longer interfere with the processing of an item (Scullin et al., 2009; see also the related work by Marsh et al., 1998; Marsh et al., 1999). In sum, many data indicate that once a prospective response is completed the corresponding intention is deactivated, and consequently, 
there is little remaining prepotent response to cause a commission error by contributing to the incorrect performance of the action on a subsequent task.

However, in contrast to the foregoing data, Scullin, Bugg, and McDaniel (2012) showed that completed intentions can contribute to prospective memory commission errors on a subsequent task. In their study, older and younger adults completed an ongoing task in Phase 1 that required a prospective response, and in Phase 2 they completed a lexicaldecision task. Even though the lexical-decision task in Phase 2 required no prospective response, Scullin et al. (2012) observed that under certain conditions, participants-especially older adults - were vulnerable to making prospective memory commission errors on this task to words that had been associated with a prospective response in the previous phase. Interestingly, these commission errors were particularly common in older adults with diminished inhibitory executive abilities.

Overall, then, existing research suggests opposite conclusions about the effects of completing an intention on subsequent vulnerability to incorrectly completing the intention again: Either completed intentions are deactivated and should not contribute to commission errors, or completed intentions can contribute to commission errors, at least in older adults. Building on the findings of Scullin et al. (2012), we predict that younger adults should be especially vulnerable to committing prospective memory commission errors when (a) their attention is distracted and (b) they encounter prospective cues that have been associated with a habitual response. We hypothesize that prospective actions that have been performed repeatedly remain in an active state, even when the actions are completed, and that attentional resources are needed to suppress performing habitual prospective responses when they are no longer necessary.

We used a prospective memory paradigm in which participants completed a lexical-decision task (i.e., making word/nonword judgments) and made a prospective response whenever they encountered a designated prospective memory cue (see, e.g., Marsh et al., 2002). An initial habit/nohabit phase was designed to either create or not create habitual prospective responses. During this phase, participants encountered prospective memory cues either multiple times (habit condition) or one time only (no-habit condition). In a subsequent test phase, we examined the effect of these formerly habitual or nonhabitual prospective responses on performance. Participants were given a new set of prospective cues to respond to during the test phase. However, the critical items during this phase were ones that had been associated with a prospective response during the previous habit/no-habit phase but that required no such prospective response during the test phase. Moreover, this test phase was completed with either full or divided attention.
We predicted that dividing attention, relative to full attention, would increase commission errors in the habit condition but not in the no-habit condition, because the reduced attentional resources would be insufficient to inhibit making a prepotent response. We conducted two different experiments, which we will refer to as Experiments $1 \mathrm{~A}$ and $1 \mathrm{~B}$, to test this prediction.

\section{Method}

\section{Participants and design}

The participants were 96 individuals in Experiment 1A and 96 individuals in Experiment 1B. All of the participants were undergraduate students at the University of Virginia and received either course credit or payment for their participation. ${ }^{1}$ Each participant was assigned to one of four groups, created in our 2 (habit vs. no-habit) $\times 2$ (full vs. divided attention) between-participants design.

\section{Materials and procedure}

Experiments 1A and 1B were identical in all respects, except for the colors used for the items and the background. In Experiment 1A, the items (i.e., words and nonwords) were shown in a black font on a white background, whereas in Experiment 1B, the items were shown in a white font on a black background.

There were three phases in the experiment: (1) a lexicaldecision familiarization task, (2) a habit/no-habit creation phase, and (3) a test phase. Following Marsh et al. (2002), words and nonwords for the lexical-decision task in all phases were drawn from a pool of medium- to highfrequency words, ranging from three to ten letters and from one to four syllables. Nonwords were created by changing one or two consonants, while keeping each nonword pronounceable (e.g., sprink). For the habit/no-habit and test phases, prospective cues and their controls were also taken from this pool and were matched on frequency $(M=138)$, number of letters (range: 4-7), and number of syllables (range: 1-2). The cues and their controls were counterbalanced across experimental conditions.

We first familiarized participants with the lexicaldecision task by having them complete 30 practice trials

\footnotetext{
${ }^{1}$ In Experiment $1 \mathrm{~A}$, the data from seven individuals were not analyzed because (a) six individuals failed to follow the instructions by not making a prospective memory response to any cues in either the habit/no-habit phase or the test phase, and (b) the distractor task malfunctioned for one individual. The data from seven individuals were not analyzed for Experiment 1B because these individuals failed to follow instructions by not making a prospective memory response to any cues during either the habit/no-habit phase or the test phase.
} 
(15 words and 15 nonwords), during which they were instructed to press the " $\mathrm{j}$ " key when a word was presented and to press the "f" key when a nonword was presented. After each word/nonword judgment, the participants saw a message that said "Waiting ..." and were instructed to press the space bar during the message to go to the next trial.

The habit/no-habit creation phase served the purpose of either creating or not creating a habitual prospective response. This phase used a prospective memory paradigm in which a lexical-decision task was performed with an embedded prospective memory task. The participants were instructed that the experimenters were interested in people's ability to remember to perform an action in the future. Participants were given a list of eight words to memorize and were told that whenever one of those words was encountered during the lexical-decision task, they were to press the Shift key after making the word/nonword judgment (i.e., first indicate that an item is a word by pressing the " $\mathrm{j}$ " key, then press the Shift key). Participants were given 2 min to memorize the eight prospective cues, and they could not begin this phase until they had correctly recalled all eight prospective memory cues. Following Marsh et al. (2002), participants then completed a filler task for $5 \mathrm{~min}$ before beginning the habit/no-habit phase.

The lexical-decision component of this habit/no-habit phase consisted of a mixture of 200 words and 200 nonwords. Participants in the no-habit condition encountered each of the eight cue words once only, with an interval of 35 trials between them, beginning at the twentieth trial. Eight control words were presented ten trials after their respective cues. In the habit condition, the eight cue words were encountered ten different times, under the constraint that (a) the cue words were randomly intermixed and evenly distributed throughout the task, and (b) a minimum of four trials were interposed between the cues. The eight control words were also encountered ten different times, each of which was presented a minimum of one trial away from its respective cue word. Because control words are matched in nearly all respects to the cue words, responses to both kinds of words would let us examine the consequences of forming an intention and responding to the cue words. Upon completion of this phase, participants freely recalled the cue words.

Participants began the test phase by learning that they were going to receive another list of eight words to memorize for a task similar to the one that they had just completed. Four of the eight cues were repeat cues, because these cues had been part of the set of cues during the previous habit/nohabit phase. The remaining four cues were novel cues, because they had not been encountered before in the experiment. Participants were provided 2 min in which to memorize the eight cues, and they could not proceed until they had recalled all eight cues correctly.
We emphasized to the participants that they should only make prospective responses to the just-memorized set of eight cue words. Participants then estimated how confident they were that they would make the appropriate prospective responses to the eight cue words on the upcoming task by using a scale from 0 (guessing) to 100 (certain). Participants then completed a 5-min filler task before beginning the test phase.

The test phase also consisted of a combination lexicaldecision and prospective memory task. The lexical-decision task consisted of a different set of 200 words and 200 nonwords than those that had been used in the habit/no-habit phase. In addition, there were eight prospective cues (i.e., four repeat and four novel cues). However, the critical items in this phase were four irrelevant cues that referred to items that had been prospective cues during the previous habit/nohabit phase but that were not prospective cues for the test phase. These four irrelevant cue items served the purpose of measuring whether participants would mistakenly make a prospective response when it is no longer necessary to do so. Finally, each of the foregoing types of cues was presented once only, along with its respective control-matched words. Each of the cue words was presented 25 trials from the others, beginning at Trial 30. A matched control word was presented ten trials after its respective cue.

Finally, our divided-attention manipulation only occurred during the test phase. Participants in the full-attention condition were told that they were going to complete a task that was similar to the one that they had just completed. Participants in the divided-attention condition were instructed that in addition to performing a task that was similar to the one that they had completed, they were going to perform a digit-monitoring task (e.g., Jacoby, 1991). Participants listened to a series of digits, presented at a rate of one digit every $2 \mathrm{~s}$, and indicated the occurrence of three consecutive odd digits by saying "Now!" After completing the test phase, the participants recalled the words that had served as prospective cues for this phase.

\section{Results}

The primary aim was to examine prospective memory commission errors during the test phase. Would the participants be vulnerable to performing prospective responses that were no longer required, and would this vulnerability increase with divided attention and for previously habitual prospective responses? In the subsequent analyses, we were interested in prospective memory performance that was influenced by memory for the intention and not by failing to remember the cue word. Consequently, we examined the proportions of correctly recalled cue words that were associated with a prospective response. 
Habit/no-habit creation phase

At the end of the habit/no-habit phase, participants in Experiment $1 \mathrm{~A}$ tended to recall more prospective cues when they were in the habit than in the no-habit condition, and in Experiment 1B the recall rates were similar for both conditions [in Exp. 1A, $82 \%$ vs. $70 \%, t(87)=2.62, p<.05, d=$ 0.56 ; in Exp. $1 \mathrm{~B}, 82 \%$ vs. $83 \%, t(87)<1]$. Prospective memory accuracy (i.e., the proportion of correctly recalled cues that were associated with a correct prospective response) was better in the habit condition, when the participants had encountered cues repeatedly, than in the no-habit condition, when the participants had encountered each cue one time only [in Exp. 1A, $88 \%$ vs. $78 \%$, $t(86)=2.41, p<.05, d=0.53$; in Exp. 1B, $92 \%$ vs. $85 \%, t(87)=2.39, p<.05, d=0.56]^{2}$

\section{Test phase}

Before beginning the test phase, participants provided a confidence rating about their expected level of success in making prospective responses. There were no differences between the habit and no-habit conditions in either Experiment 1A (ratings of 71 vs. 68, respectively), $t<1$, or Experiment 1B (ratings of 76 vs. 75, respectively), $t<1$. Moreover, in the divided-attention conditions, we found no significant differences in digit-monitoring accuracy between the habit and no-habit conditions in Experiment 1A (80\% vs. $78 \%$, respectively), $t<1$, and Experiment $1 \mathrm{~B}$ ( $86 \%$ vs. $79 \%$, respectively), $t<1.73$, indicating comparable distractibility in both conditions.

Our central hypothesis concerned the ability to withhold making a prospective response. We examined commission errors to those irrelevant cues that an individual had correctly recalled at the end of the habit/no-habit phase, shown in Fig. 1 for both Experiments 1A and 1B. Both experiments show identical patterns: more commission errors in the habit divided-attention condition than in any of the other conditions.

We set out to test one hypothesis with our design: that dividing attention, relative to full attention, would increase commission errors in the habit condition but not in the nohabit condition. Given that we were predicting one specific pattern in the data, many statisticians (e.g., Rosnow \& Rosenthal, 1996; Rosenthal \& Rosnow, 1985) have noted that focused contrasts are a more appropriate and powerful method of testing a priori hypotheses than are unfocused omnibus ANOVAs followed by post-hoc tests. Using contrast weights of $3,-1,-1,-1$ for our habit divided, habit full, no-habit divided, and no-habit full conditions, respectively,

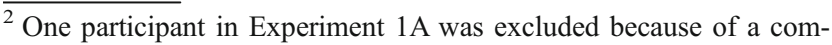
puter error.
}
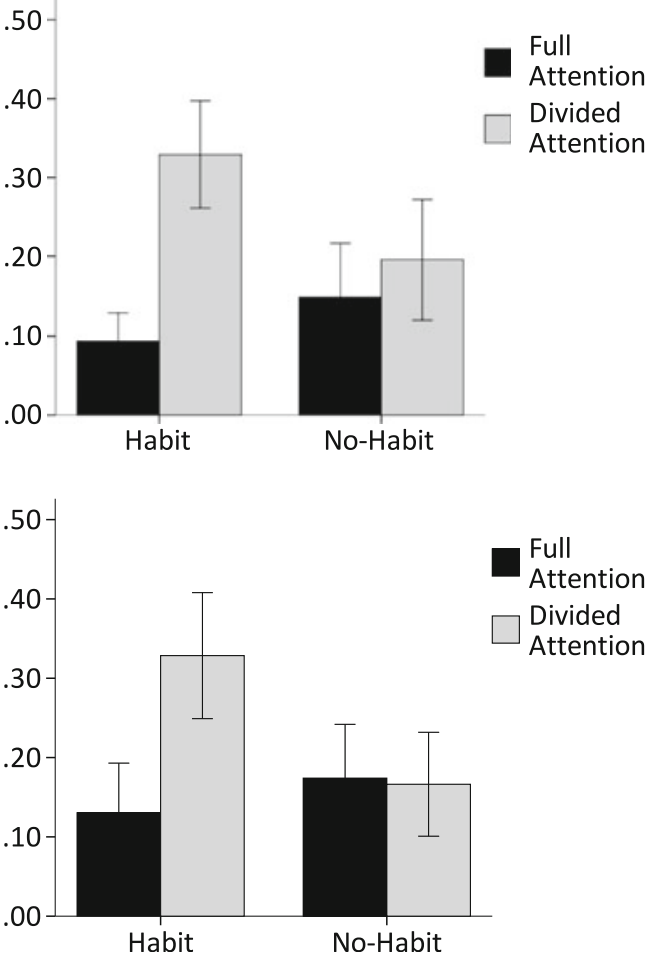

Fig. 1 Proportions of cues recalled at the end of the habit/no-habit phase that were subsequently associated with a commission error during the test phase, for Experiments 1A (top panel) and 1B (bottom panel)

we conducted a focused contrast to test the presence of this predicted pattern in the data. In Experiment $1 \mathrm{~A}$, this contrast was significant, $F(1,81)=6.53, M S E=.08$, $p<.05, \eta_{\mathrm{p}}^{2}=.07$. Replicating Experiment 1A, Experiment 1B also showed a significant contrast, $F(1,83)=4.72, M S E=.10$, $p<.05, \eta_{\mathrm{p}}^{2}=.05$. Overall, these analyses confirmed what is clear in Fig. 1: Commission errors to irrelevant cues increased when participants both were distracted and encountered irrelevant cues that had been associated with a habitual prospective response.

Is it possible that the increased commission errors in the habit divided-attention group were caused by a tendency to respond to familiar items in general, as opposed to our hypothesis that participants were responding to cues that had been associated with a habitual intention? To rule out this familiarity account, we examined the rate of commission errors to the control items for these irrelevant cues. As a reminder, these control items also appeared during the habit/ no-habit phase and during the test phase and were also matched to the irrelevant cues in all dimensions, except that the control items (a) were not associated with an intention and (b) had not been learned perfectly at the beginning of the experiment. If familiarity compelled a response when attention was divided, we should observe more commission errors to control items in the habit than in the no-habit 
conditions (i.e., where these control items were encountered ten times vs. one time, respectively). Commission errors to these control items were essentially nonexistent in both experiments, as there were no errors to any of these items for nearly all participants, except for one individual in the no-habit full-attention condition of Experiment 1A, and four individuals in Experiment 1B (two in the no-habit full-attention condition, one in the no-habit divided-attention condition, and one in the habit full-attention condition). Thus, there is no support for the account that individuals generally responded to items that were relatively more familiar than others. Instead, these commission errors occurred to cues that were associated with a habitual intention under conditions of cognitive distraction.

Before describing correct prospective memory performance, we will assess the proportions of novel and repeat prospective cues that were correctly recalled at the end of the test phase with a 2 (cue type: novel vs. repeat cue) $\times 2$ (condition: habit vs. no habit) $\times 2$ (attention: full vs. divided) ANOVA. ${ }^{3}$ In Experiment $1 \mathrm{~A}$, there were no significant main effects or interactions, all $F \mathrm{~s}<1.76$. Table 1 shows that recall performance was comparable across all of the conditions. In Experiment 1B, however, this three-way ANOVA yielded a significant interaction between cue type and condition, $F(1,82)=6.26, M S E=.03, p<.05, \eta_{\mathrm{p}}^{2}=.07$, which was qualified by a three-way interaction, $F(1,82)=$ $8.37, M S E=.03, p<.01, \eta_{\mathrm{p}}^{2}=.09$. As is shown in Table 1, the latter interaction reflects the fact that recall performance in Experiment 1B was higher for repeat than for novel cues in all conditions except the habit divided condition, which showed the opposite pattern. We also investigated the rate at which participants incorrectly intruded items from the habit/no-habit phase when recalling cues from the test phase. The proportions of target items from the habit/no-habit phase that were incorrectly recalled on this test were low and comparable across the habit conditions $\left(M_{\text {Full }}=.03, M_{\text {Div }}=.07\right.$ in Exp. 1A; $M_{\text {Full }}=.00, M_{\text {Div }}=.06$ in Exp. 1B) and the no-habit conditions $\left(M_{\text {Full }}=.02, M_{\text {Div }}=.06\right.$ in Exp. $1 \mathrm{~A} ; M_{\text {Full }}=.01, M_{\text {Div }}=.02$ in Exp. 1B). A 2 (habit vs. no habit) $\times 2$ (full vs. divided) ANOVA of these intrusions yielded no significant main effects or interactions for either experiment, all $F_{\mathrm{S}}<2.38$ for Experiment 1A, and all $F_{\mathrm{s}}<3.86$ for Experiment 1B.

With respect to how well participants correctly made prospective responses, Table 1 presents the proportions of correctly recalled novel and repeat cues that were associated

\footnotetext{
${ }^{3}$ Due to experimenter error, recall data were not collected from three individuals in the habit divided-attention condition and from four individuals in the no-habit divided-attention condition in Experiment $1 \mathrm{~A}$, and from one individual in the habit divided condition and two individuals in the no-habit divided condition in Experiment 1B.
}

with a correct prospective response. ${ }^{4}$ For Experiment 1A, a 2 (habit vs. no habit) $\times 2$ (full vs. divided attention) $\times 2$ (novel vs. repeat cue) ANOVA of the proportions of correct responses showed a main effect of attention, $F(1,77)=$ 19.76, MSE $=.11, p<.001, \eta_{\mathrm{p}}^{2}=.20$, with better accuracy under full attention (.77) than under divided attention (.54). We also found a main effect of type of cue, $F(1,77)=15.78$, $M S E=.06, p<.001, \eta_{\mathrm{p}}^{2}=.17$, whereby prospective memory accuracy was better for repeat cues (.75) than for novel cues (.60). There were no other significant effects, all $F_{\mathrm{S}}<$ 3.09. Likewise, the results from Experiment $1 \mathrm{~B}$ showed a similar pattern: a 2 (habit vs. no habit) $\times 2$ (full vs. divided attention) $\times 2$ (novel vs. repeat cue) ANOVA of correct conditional prospective responses showed a main effect of attention, $F(1,79)=17.51, M S E=.12, p<.001, \eta_{\mathrm{p}}^{2}=.18$, with better accuracy under full attention (.79) than under divided attention (.57). A main effect of the type of cue also emerged, $F(1,79)=40.31, M S E=.06, p<.001, \eta_{\mathrm{p}}^{2}=.34$, with greater accuracy for repeat cues (.81) than for novel cues (.57). However, the foregoing two effects were qualified by an Attention $\times$ Cue interaction, $F(1,79)=$ $4.49, M S E=.06, p<.05, \eta_{\mathrm{p}}^{2}=.05$, whereby divided attention exerted a greater cost on responses to novel cues (full $=.71$ vs. divided $=.41)$ than it did on responses to repeated cues (full $=.87$ vs. divided $=.73$ ). No other significant effects were found, all $F_{\mathrm{S}}<1.78$. Overall, both experiments showed similar patterns: Prospective memory accuracy was better under full than under divided attention, and it was better with cues that had been responded to before (repeat cues) than with novel cues.

\section{Discussion}

After routinely responding to prospective cues during a habit/no-habit phase, participants were vulnerable on a subsequent test to commission errors when their attentional resources were taxed. Our experimental paradigm was analogous to the situation in which an individual must continue taking some medication but no longer needs to take other medications, such as seasonal allergy medication. In this situation, seeing the allergy medication will trigger a response that may not be suppressed when the individual is distracted. Our data ruled out a familiarity account and showed that participants did not just commit prospective memory errors to all familiar items during the test phase. Specifically, control items [i.e., items matched to the prospective cue items in all respects, except (a) for the absence of an intention to respond and (b) that these items had not

\footnotetext{
${ }^{4}$ One individual in Experiment $1 \mathrm{~A}$ and three individuals in Experiment $1 \mathrm{~B}$ failed to recall any novel or repeat cues, and therefore could not contribute data to these analyses.
} 
Table 1 Recall performance and correct prospective responses to the novel and repeat cues

\begin{tabular}{|c|c|c|c|c|c|c|c|c|}
\hline \multirow[t]{3}{*}{ Condition } & \multicolumn{4}{|c|}{ Experiment 1A } & \multicolumn{4}{|c|}{ Experiment 1B } \\
\hline & \multicolumn{2}{|l|}{ Test Recall } & \multicolumn{2}{|c|}{ Prospective Accuracy } & \multicolumn{2}{|l|}{ Test Recall } & \multicolumn{2}{|c|}{ Prospective Accuracy } \\
\hline & $\begin{array}{l}\text { Novel Cue } \\
M(S E)\end{array}$ & $\begin{array}{l}\text { Repeat Cue } \\
M(S E)\end{array}$ & $\begin{array}{l}\text { Novel Cue } \\
M(S E)\end{array}$ & $\begin{array}{l}\text { Repeat Cue } \\
M(S E)\end{array}$ & $\begin{array}{l}\text { Novel Cue } \\
M(S E)\end{array}$ & $\begin{array}{l}\text { Repeat Cue } \\
M(S E)\end{array}$ & $\begin{array}{l}\text { Novel Cue } \\
M(S E)\end{array}$ & $\begin{array}{l}\text { Repeat Cue } \\
M(S E)\end{array}$ \\
\hline Habit full-attention & $.82(.05)$ & $.86(.05)$ & $.64(.07)$ & $.81(.06)$ & $.85(.05)$ & $.92(.04)$ & $.69(.06)$ & $.85(.05)$ \\
\hline Habit divided-attention & $.80(.06)$ & $.80(.06)$ & $.39(.07)$ & $.68(.05)$ & $.87(.05)$ & $.72(.08)$ & $.46(.08)$ & $.68(.08)$ \\
\hline No-habit full-attention & $.80(.05)$ & $.86(.05)$ & $.80(.06)$ & $.86(.04)$ & $.83(.05)$ & $.88(.05)$ & $.73(.07)$ & $.89(.03)$ \\
\hline No-habit divided-attention & $.88(.06)$ & $.85(.06)$ & $.49(.08)$ & $.61(.10)$ & $.77(.06)$ & $.93(.04)$ & $.36(.08)$ & $.77(.06)$ \\
\hline
\end{tabular}

Recall occurred at the end of the test phase. Prospective accuracy refers to the proportions of correctly recalled cues that were associated with a correct prospective response during the test phase. Parentheses contain the standard errors of the means.

been learned perfectly in Phase 1] were encountered ten different times by individuals in the habit group during the initial habit/no-habit phase, as compared to just one time for those in the no-habit group. Yet the error rates for these items during the test phase were close to zero for both groups - and numerically lower for the habit group - and dividing attention had no effect on error rates.

In sum, individuals make prospective memory errors to items under the combination of three conditions: (1) The items are associated with an intention; (2) the intention has been performed many times; and (3) the individuals are distracted. Together, these data suggest that inhibitory failures cause prospective memory errors and that cognitive control processes are required to prevent making a previously habitual prospective memory response.

It is important to consider alternative explanations for this increased rate of commission errors on the part of individuals in the habit group. According to the preparatory and attentional processes (PAM) model (Smith, 2003; Smith \& Bayen, 2004), dividing attention should challenge the process of recognizing valid prospective cues, which in turn would lower hit rates to valid cues and raise false alarm rates to invalid cues. One difficulty for this account is that we did not observe that dividing attention increased false alarm rates overall, as false alarm rates for control items were numerically lower-and close to zero-in the divided-attention condition than in the full-attention condition. Instead, divided attention only increased false alarms to irrelevant cues that had been responded to habitually. Similarly, a source confusion account would argue that participants make prospective memory errors and respond to irrelevant cues because they misrecollect which cues are the appropriate prospective memory cues to respond toessentially confusing previously relevant cues with the currently relevant ones. If a source confusion account were correct, at the end of the test phase, when we asked participants to recall the set of eight relevant prospective cues, we should have observed more intrusions of the critical items in the divided-attention than in the fullattention condition. This was not the case, as intrusion rates were very low in all conditions. An additional difficulty for both the PAM and source confusion accounts is that Scullin et al. (2012) showed, in a very similar paradigm, that older adults are particularly vulnerable to make prospective memory responses to previously valid cues when they complete a task that requires no prospective memory responses-a type of error that is consistent with our inhibitory-failure account and inconsistent with both the PAM and source confusion accounts.

Both Experiments $1 \mathrm{~A}$ and $1 \mathrm{~B}$ showed that commission errors occur in the no-habit conditions at rates of about $15 \%$. These data raise two questions: (1) What accounts for the nonnegligible commission error rate in this no-habit condition? and (2) How come dividing attention does not increase commission errors in the no-habit condition? Our data suggest that responding to a prospective cue just one time only during Phase 1 (i.e., the no-habit condition) is sufficient to activate the cue, so that participants will occasionally false alarm to this irrelevant cue during the subsequent test phase, producing a commission error. However, speculatively, the level of activation of the irrelevant cues in the nohabit condition was too low to produce a substantial increase when attention was divided.

Our findings point out a boundary condition to the conclusion that finished intentions are deactivated (e.g., Marsh et al., 1999; Scullin et al., 2009). As we reviewed earlier, mounting research has indicated that nonhabitual intentions are deactivated when they are completed, as is shown by the diminished intention interference effect for finished, as compared to active, intentions. By contrast, our data indicate that habitual intentions remain in an active state, even when they are finished, and can be erroneously performed at a later time when an individual is distracted. 
Author note We are very appreciative of the helpful comments of Lia Kvavilashvili, Jason Hicks, and the other participants of ICOM 5 (International Conference on Memory).

\section{References}

Cohen, A.-L., Dixon, R. A., \& Lindsay, D. S. (2005). The intention interference effect and aging: Similar magnitude of effects for young and old adults. Applied Cognitive Psychology, 19, 11771197.

Einstein, G. O., \& McDaniel, M. A. (1990). Normal aging and prospective memory. Journal of Experimental Psychology. Learning, Memory, and Cognition, 16, 717-726.

Einstein, G. O., McDaniel, M. A., Thomas, R., Mayfield, S., Shank, H., Morrisette, N., \& Breneiser, J. (2005). Multiple processes in prospective memory retrieval: Factors determining monitoring versus spontaneous retrieval. Journal of Experimental Psychology. General, 134, 327-342. doi:10.1037/0096-3445.134.3.327

Jacoby, L. L. (1991). A process dissociation framework: Separating automatic from intentional uses of memory. Journal of Memory and Language, 30, 513-541. doi:10.1016/0749-596X (91)90025-F

Marsh, R. L., Hicks, J. L., \& Bink, M. L. (1998). Activation of completed, uncompleted, and partially completed intentions. Journal of Experimental Psychology. Learning, Memory, and Cognition, 24, 350-361.
Marsh, R. L., Hicks, J. L., \& Bryan, E. S. (1999). The activation of unrelated and canceled intentions. Memory and Cognition, 27, $320-327$.

Marsh, R. L., Hicks, J. L., \& Watson, V. (2002). The dynamics of intention retrieval and coordination of action in event-based prospective memory. Journal of Experimental Psychology. Learning, Memory, and Cognition, 28, 652-659.

Rosenthal, R., \& Rosnow, R. L. (1985). Contrast analysis: Focused comparisons in the analysis of variance. New York: Cambridge University Press.

Rosnow, R. L., \& Rosenthal, R. (1996). Computing contrasts, effect sizes, and counternulls on other people's published data: General procedures for research consumers. Psychological Methods, 1, 331-340.

Scullin, M. K., Bugg, J. M., \& McDaniel, M. A. (2012). Whoops, I did it again: Commission errors in prospective memory. Psychology and Aging, 27, 46-53. doi:10.1037/a0026112

Scullin, M. K., Einstein, G. O., \& McDaniel, M. A. (2009). Evidence for spontaneous retrieval of suspended but not finished prospective memories. Memory and Cognition, 37, 425-433. doi:10.3758/MC.37.4.425

Smith, R. E. (2003). The cost of remembering to remember in eventbased prospective memory: Investigating the capacity demands of delayed intention performance. Journal of Experimental Psychology. Learning, Memory, and Cognition, 29, 347-361.

Smith, R. E., \& Bayen, U. J. (2004). A multinomial model of eventbased prospective memory. Journal of Experimental Psychology. Learning, Memory, and Cognition, 30, 756-777. doi:10.1037/ 0278-7393.30.4.756 